\title{
The antioxidants dilemma: are they potentially immunosuppressants and carcinogens?
}

\author{
Soroush Seifirad $^{1 *}$, Alireza Ghaffari ${ }^{2}$ and Mahsa M. Amoli ${ }^{1}$ \\ ${ }_{1}^{1}$ Endocrinology and Metabolism Research Center, Endocrinology and Metabolism Clinical Sciences Institute, Tehran University of Medical Sciences, Tehran, Iran \\ ${ }^{2}$ Department of Internal Medicine, Tabriz University of Medical Sciences, Tabriz, Iran \\ *Correspondence: sseifirad@gmail.com
}

Edited and reviewed by:

Sruti Shiva, University of Pittsburgh, USA

Keywords: antioxidants, carcinogens, protective agents, immunosuppressive agents, reactive oxygen species, DNA damage, modeling

\section{INTRODUCTION}

Antioxidants and a large number of natural supplements were introduced in recent decades (Lu et al., 2013). It has been hypothesized that antioxidant consumption might reverse oxidative stress which decreases the adverse effects elicited as outcomes of oxidative stress including inflammation, carcinogenesis, and atherosclerosis (Ghorbanihaghjo et al., 2012; Bjelakovic et al., 2014). A large number of studies have been conducted in order to convey evidence for beneficial effects of antioxidants in health and disease (Bjelakovic et al., 2012). However, most of these studies were conducted for a short time interval evaluating limited numbers of targeted biochemical markers both in animal and human studies. Only a small number of prolonged cohort studies have been performed to critically assess the effects of chronic consumption of antioxidants. Unexpectedly, in a recently published systematic review by Bjelakovic et al. on 78 randomized clinical trials on antioxidants supplementation including selenium, $\beta$-carotene, vitamin $C$, vitamin $A$, and vitamin $E$, not only have no favorable effects been observed, but additionally, mortality rates have risen (Bjelakovic and Gluud, 2007; Bjelakovic et al., 2012). Surprisingly, it has been shown that antioxidant supplementation may increase the risk of skin malignancy in women (Hercberg et al., 2007). There are also a number of reports, in which a history of longtime supplementation with carotenoids has increased risk of malignancy in smokers and patients with tuberculosis (Albanes et al., 1996; Omenn et al., 1996; Holick et al., 2002; Shiels et al., 2011).
Natural and green products are assumed to be harmless in common belief. The general faith toward the health benefits of products containing antioxidants is partly because of misinformation conveyed by industries manufacturing these food products, which promoted putative overuse of these products as dietary supplements. However, the harmful effects of these products have remained obscured for unknown reasons (Lu et al., 2013). It is crucial to emphasize the harmful effects of the chronic consumption of antioxidants, including potential toxicity, in addition of potential benefits. According to Watson "the time has come to seriously ask whether antioxidant use much more likely causes than prevents cancer" (Watson, 2013). In this article we have tried to discuss probable mechanisms by which chronic antioxidant consumption provokes adverse effects. We have attempted to depict the role of antioxidants in a comprehensive model of inflammation, oxidative stress, and cancer.

\section{NATURAL OXIDATIVE PATHWAYS}

From an evolutionary point of view, oxygen was a toxic component which turned into one of the most important molecules essential for life and a vital agent after millions of years of evolution. However, in certain conditions, its toxic effects still continue to protect more evolved organisms against certain pathogens. Reactive oxygen species (ROS) which play an important role in the innate immune response are a group of molecules and reactive ions and radicals derived from oxygen. Phagocytes mainly comprised of macrophages and neutrophils release a variety of molecules including toxic oxygen radicals in a process undergoing respiratory burst upon activation in response to infectious agents and pathogens. It has been observed that patients with impaired ROS production are critically immune suppressed (i.e., patients with chronic granulomatosis disease); (Segal et al., 2000; Assari, 2006) Therefore, it can be assumed that oxidative stress does not always incite damaging effects. Toxic oxygen-derived products such as superoxide, hydrogen peroxide, singlet oxygen, and hydroxyl radical play a critical role in oxygen-dependent intracellular killing of pathogens, and hence, are key factors within the immune system.

\section{OXIDATIVE STRESS: A DOUBLE-EDGED SWORD}

ROS could be toxic to both normal and abnormal cells (infected by intracellular pathogens and malignant cells). It has been shown that increased oxidative stress could enhance prevalence of malignancies by direct cellular damage, Seifirad et al. (2012); Lu et al. (2013); Seifirad and Masoudkabir (2013) however, as mentioned above oxidative stress when applied as immune system arms could protect organisms from invading pathogens and malignant cells (Weel et al., 1996).

\section{REDUCTIVE STRESS: A FORGOTTEN ENEMY}

Reductive stress concept has been recently introduced. It seems that although reducing agents provoke many adverse effects and cause damages, this phenomenon has been obscured in the shadow of oxidative stress. Antioxidants could also be categorized as reducing agents.

Antioxidants are rich in weakly-bound electrons and could cause direct DNA damage (Lu et al., 2013). Wang and 
Nguyen, and their research team have demonstrated that the dissociative electron transfer (DET) of weakly-bound $\mathrm{e}_{\text {pre }^{-}}$ to the guanine base is extremely efficient in inducing chemical bond breaks and subsequent breaking of single and double strand DNA in aqueous solutions (Wang et al., 2009; Nguyen et al., 2011).

It should be noted that the superoxide anion $\left(\mathrm{O}_{2^{-}}\right)$would act as an oxidant and a strong reducing agent. In fact $\mathrm{O}_{2}$ has a distinguished electron affinity since $\mathrm{O}_{2^{-}}$has no positive electron affinity ( $\mathrm{Lu}$ et al., 2013). In aqueous media $\mathrm{O}_{2^{-}}$act as a strong Bronsted base and donates an electron (i.e., Fenton reaction) (Bhattacharjee et al., 2012; Enami et al., 2014). Hence, $\mathrm{O}_{2-}$ could induce reductive damage to the cell and DNA (Lu et al., 2013).

Recent studies demonstrate that ROS detoxication and enhanced intracellular antioxidant might be pro-tumorigenic
(DeNicola et al., 2011; Perera and Bardeesy, 2011). It has been shown that high intake of tea or coffee that are rich in flavonoids in pregnant women might increase the risk of central nervous system tumors and childhood leukemia (Strick et al., 2000; Paolini et al., 2003; Plichart et al., 2008).

In a very recently published study, $\mathrm{Lu}$ et al. showed that natural antioxidant supplements may cause adverse effects in healthy humans, and they may increase the rate of malignancies rather than preventing cancers ( $\mathrm{Lu}$ et al., 2013). By means of femtomedicine, they compared the toxic effects of green tea extract (GT) epigallocatechin gallate (EGCG) as the main flavonoid in green tea, $\mathrm{H}_{2} \mathrm{O}_{2}$, and Cisplatin on human lung and skin normal cells. Based on the $\mathrm{IC}_{50}$ values (concentration required to kill $50 \%$ of untreated cells), they showed that that EGCG and GT were definitely highly toxic against human skin and lung normal cell and treatment with EGCG decreased the cell survival rate in a dose dependent manner. Surprisingly, they also demonstrated that while both EGCG and $\mathrm{O}_{2}{ }^{-}$have a similar redox potential, the reductive damage induced by $\mathrm{O}_{2}{ }^{-}$must be much less than EGCG. According to their results, treatment with EGCG/GT slightly increased the survival rate of lung cancer cells at low concentrations (\#100/150 mM), despite the fact that treatment with very high concentrations (100-400 or $150-500 \mathrm{mM}$ ) showed some therapeutic effects. This observation was also in accordance with the previous findings (Yang et al., 1998, 2000; Elbling et al., 2005). It should be noted that although extremely high levels of EGCG/TG (100$500 \mathrm{mM}$ ) can destroy cancerous cells via the reductive mechanism and DNA damage induction, normal cells are also

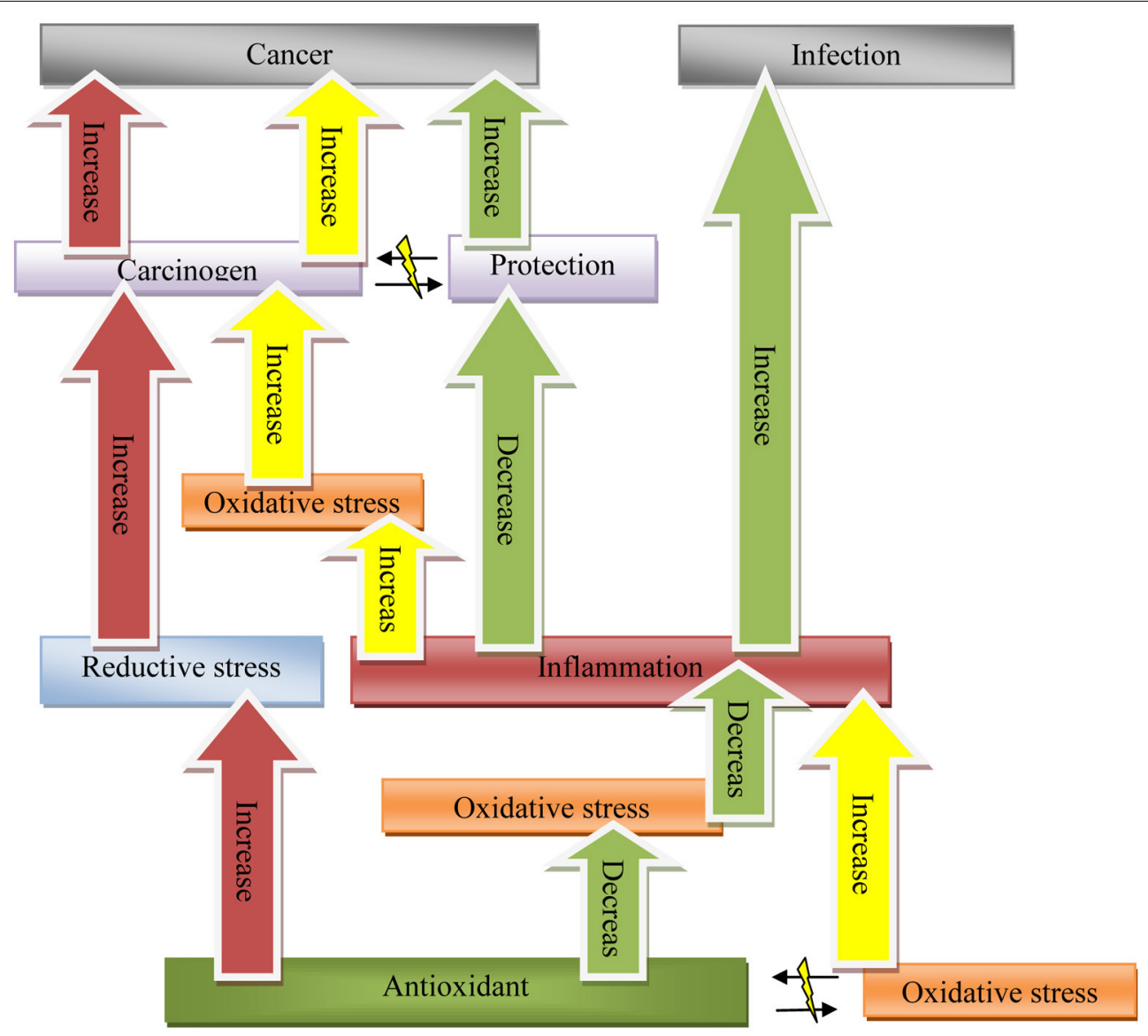

FIGURE 1 | A comprehensive model for oxidative stress, antioxidants, inflammation, and carcinogenesis: oxidant-antioxidant equilibrium, cancer protection, and carcinogenesis equilibrium are described as determinant balancing points between health and disease states in this model. Red: Antioxidants as reductive agent. Yellow: Oxidative stress carcinogenesis pathway. Green: Antioxidant as immunosuppressant. 
threatened to be severely damaged at such high level of antioxidants treatment. Therefore, it might be suggested that EGCG/antioxidant supplementation might not have beneficial effects for patients undergoing chemotherapy. In another words, antioxidants may diminish the ability of an exogenous reducing agent in killing tumor cells (Watson, 2013).

\section{ANTIOXIDANT PATHWAYS}

As it has been illustrated in Figure 1:

1. Antioxidants decrease oxidative stress and oxidative damage and subsequently diminish likelihood of developing malignancies due to oxidative stress. This pathway has been the foundation of current thoughts leading to the antioxidant general administration and usage in common belief.

2. Antioxidants could impair function of immune system by means of decreased oxidative stress. This might increase prevalence of malignancies and infections (Figure 1 following green pathway).

3. Since antioxidants are rich in weaklybound electrons, they could cause direct DNA and cell damage. Hence they could be carcinogens (Figure 1 following red pathway).

\section{EOUILIBRIUM AND HOMEOSTASIS}

Traditionally disequilibrium in natural homeostasis is defined as disease state. In traditional medicine, diagnosis and treatment of disorders was based on managing equilibrium (Shahabi et al., 2008). Therefore, when there is equilibrium between oxidant/antioxidant pathways maximum protection is anticipated (health state). Disequilibrium in these pathways could impair defense mechanism and increase likelihood of developing malignancies and infections (Figure 2).
Here we demonstrate some examples to further discuss the effect of imbalance in antioxidant equilibrium in various conditions.

\section{SMOKING, TUBERCULOSIS, AND ANTIOXIDANTS}

Previous studies have observed an increased rate of lung cancer in smokers who were on long time supplementation with $\beta$-carotene as well as in patients with tuberculosis treated with carotenoids (Albanes et al., 1996; Omenn et al., 1996; Holick et al., 2002; Shiels et al., 2011). First of all, $\beta$-carotene in high levels and chronic consumption could be carcinogenic by means of direct DNA damage; additionally, it might be hypothesized that a slightly increased level of oxidative stress could be protective in smokers and patients with tuberculosis, while a very large increase in oxidative stress could be carcinogenic rather than protective. In fact, it seems

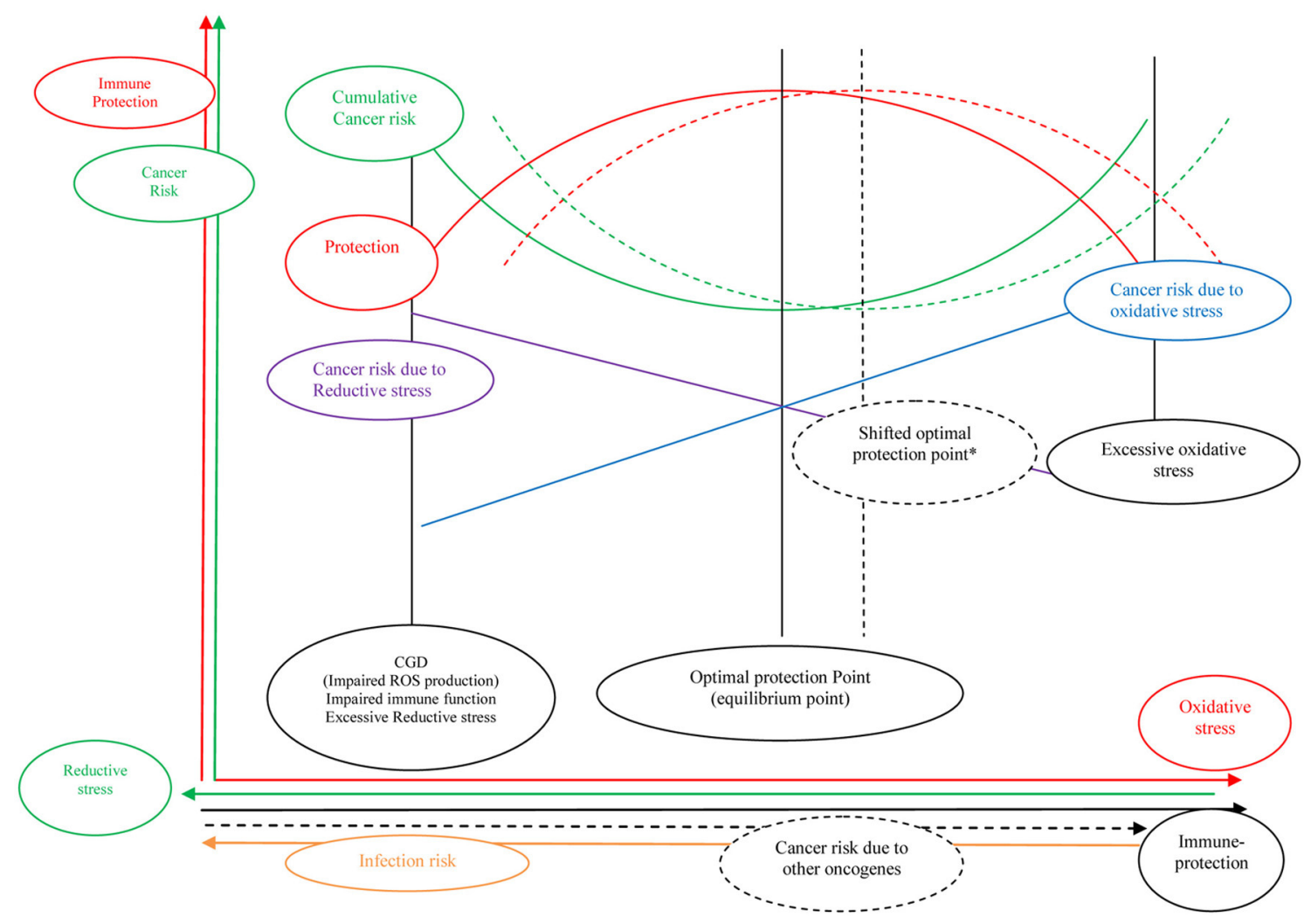

FIGURE 2 | There is no unit and all figures are representing increase and decrease in parameters, curves, and arrows are representing hypothetical relationship between parameters. *Both high levels of ROS and antioxidants are carcinogen; however it seems that in certain conditions such as tuberculosis and smoking slightly increased ROS production could be beneficial rather than hazardous. Optimal equilibrium point is shifted in these conditions to the right. Increased risk of cancer due to other oncogenes (i.e., smoking) needs more alert immune system and more oxidative stress, hence optimal protection point will shift to the right. 
that in these cases, the optimal point of oxidative stress is enhanced in order to compensate for extrinsic effects of carcinogens or pathogens (Shifted optimal protection point, Figure 2). Taken together while alert immune system could be protective, antioxidants might downregulate immune system, by means of decreased inflammation and cell damage.

\section{DISCUSSION}

Industrialization in the recent century significantly affected the human lifestyle. As the transformation in lifestyle and habits has been so rapid, it has not been attenuated as a matter of evolution by means of time. Modern human beings began smoking, as well as producing and consuming food colorings, food preservatives, and fast foods. Medicine has been developed and a large number of antibiotics and oxidant agents (i.e., alkaline agents, antibiotics, and other chemotherapy agents for anticancer treatment) were produced. On the other hand, there has been an effort by scientists to transverse these effects by exogenous artificial manipulations and interventions like antioxidant supplementation. However, the limited knowledge of complex physiology of the human system has resulted in further complications (Seifirad, 2014). Lack of appropriate knowledge and the complexity of systems and physiological pathways has exacerbated troubles of industrialized society, which is beyond the simplified approach currently utilized in resolving complex dilemmas. Broad antioxidant treatment has been one of these manipulations that might be harmful rather than beneficial if prescribed without precise consideration. In fact, in the average human diet, there would be no need for such a high consumption of supplements including antioxidants.

In conclusion, oxidative stress and inflammation are not as harmful as it has been assumed. They are natural defense systems in the human body working against infectious diseases as well as malignancies. Oblivious antioxidant therapy could be harmful rather than beneficial for health. Excessive antioxidant therapy could impair immune system and increase likelihood of developing malignancies by means of decreased immune protection and direct cell and DNA damage.

\section{SUGGESTION}

Studies on modeling and determining optimal oxidative stress point are warranted.

\section{ACKNOWLEDGMENT}

The authors would like to acknowledge Mrs. Yasamin Jalinouszadeh for her kind contribution in preparation of this article.

\section{REFERENCES}

Albanes, D., Heinonen, O. P., Taylor, P. R., Virtamo, J., Edwards, B. K., Rautalahti, M., et al. (1996). Alpha-Tocopherol and beta-carotene supplements and lung cancer incidence in the alpha-tocopherol, beta-carotene cancer prevention study: effects of base-line characteristics and study compliance. J. Natl. Cancer Inst. 88, 1560-1570. doi: 10.1093/jnci/88.21.1560

Assari, T. (2006). Chronic granulomatous disease; fundamental stages in our understanding of CGD. Med. Immunol. 5, 4. doi: 10.1186/1476-9433-5-4

Bhattacharjee, S., Chatterjee, S., Jiang, J., Sinha, B. K., and Mason, R. P. (2012). Detection and imaging of the free radical DNA in cells-site-specific radical formation induced by Fenton chemistry and its repair in cellular DNA as seen by electron spin resonance, immuno-spin trapping and confocal microscopy. Nucleic Acids Res. 40, 5477-5486. doi: 10.1093/nar/gks180

Bjelakovic, G., and Gluud, C. (2007). Surviving antioxidant supplements. J. Natl. Cancer Inst. 99, 742-743. doi: 10.1093/jnci/djk211

Bjelakovic, G., Nikolova, D., and Gluud, C. (2014). Antioxidant supplements and mortality. Curr. Opin. Clin. Nutr. Metab. Care 17, 40-44. doi: 10.1097/MCO.0000000000000009

Bjelakovic, G., Nikolova, D., Gluud, L. L., Simonetti, R. G., and Gluud, C. (2012). Antioxidant supplements for prevention of mortality in healthy participants and patients with various diseases. Cochrane Database Syst. Rev. 3:CD007176. doi: 10.1002/14651858.CD007176.pub2

DeNicola, G. M., Karreth, F. A., Humpton, T. J., Gopinathan, A., Wei, C., Frese, K., et al. (2011). Oncogene-induced Nrf2 transcription promotes ROS detoxification and tumorigenesis. Nature 475 , 106-109. doi: 10.1038/nature10189

Elbling, L., Weiss, R. M., Teufelhofer, O., Uhl, M., Knasmueller, S., Schulte-Hermann, R., et al. (2005). Green tea extract and (-)-epigallocatechin3-gallate, the major tea catechin, exert oxidant but lack antioxidant activities. FASEB J. 19, 807-809. doi: 10.1096/fj.04-2915fje

Enami, S., Sakamoto, Y., and Colussi, A. J. (2014). Fenton chemistry at aqueous interfaces. Proc. Natl. Acad. Sci. U.S.A. 111, 623-628. doi: 10.1073/pnas.1314885111

Ghorbanihaghjo, A., Kolahi, S., Seifirad, S., Rashtchizadeh, N., Argani, H., Hajialilo, M., et al. (2012). Effect of fish oil supplements on serum paraoxonase activity in female patients with rheumatoid arthritis: a double-blind randomized controlled trial. Arch. Iran. Med. 15, 549-552.

Hercberg, S., Ezzedine, K., Guinot, C., Preziosi, P., Galan, P., Bertrais, S., et al. (2007). Antioxidant supplementation increases the risk of skin cancers in women but not in men. J. Nutr. 137, 2098-2105.

Holick, C. N., Michaud, D. S., Stolzenberg-Solomon, R., Mayne, S. T., Pietinen, P., Taylor, P. R., et al. (2002). Dietary carotenoids, serum beta-carotene, and retinol and risk of lung cancer in the alphatocopherol, beta-carotene cohort study. Am. J. Epidemiol. 156, 536-547. doi: 10.1093/aje/kwf072

Lu, L. Y., Ou, N., and Lu, Q. B. (2013). Antioxidant induces DNA damage, cell death and mutagenicity in human lung and skin normal cells. Sci. Rep. 3:3169. doi: 10.1038/srep03169

Nguyen, J., Ma, Y., Luo, T., Bristow, R. G., Jaffray, D. A., and Lu, Q. B. (2011). Direct observation of ultrafast-electron-transfer reactions unravels high effectiveness of reductive DNA damage. Proc. Natl. Acad. Sci. U.S.A. 108, 11778-11783. doi: 10.1073/pnas.1104367108

Omenn, G. S., Goodman, G. E., Thornquist, M. D., Balmes, J., Cullen, M. R., Glass, A., et al. (1996) Risk factors for lung cancer and for intervention effects in CARET, the Beta-Carotene and Retinol Efficacy Trial. J. Natl. Cancer Inst. 88, 1550-1559. doi: 10.1093/jnci/88.21.1550

Paolini, M., Sapone, A., and Valgimigli, L. (2003). Avoidance of bioflavonoid supplements during pregnancy: a pathway to infant leukemia? Mutat. Res. 527, 99-101. doi: 10.1016/S00275107(03)00057-5

Perera, R. M., and Bardeesy, N. (2011). Cancer: when antioxidants are bad. Nature 475, 43-44. doi: 10.1038/475043a

Plichart, M., Menegaux, F., Lacour, B., Hartmann, O., Frappaz, D., Doz, F., et al. (2008). Parental smoking, maternal alcohol, coffee and tea consumption during pregnancy and childhood malignant central nervous system tumours: the ESCALE study (SFCE). Eur. J. Cancer Prev. 17, 376-383. doi: 10.1097/CEJ.0b013e3282f75e6f

Segal, B. H., Leto, T. L., Gallin, J. I., Malech, H. L., and Holland, S. M. (2000). Genetic, biochemical, and clinical features of chronic granulomatous disease. Medicine 79, 170-200. doi: 10.1097/00005792 200005000-00004

Seifirad, S. (2014). An emerging need for developing new models for myocardial infarction as a chronic complex disease: lessons learnt from animal vs. human studies on cardioprotective effects of Erythropoietin in reperfused myocardium. Front. Physiol. 5, 44. doi: 10.3389/fphys.2014.00044

Seifirad, S., Keshavarz, A., Taslimi, S., Aran, S. Abbasi, H., and Ghaffari, A. (2012). Effect of pirfenidone on pulmonary fibrosis due to paraquat poisoning in rats. Clin. Toxicol. 50, 754-758. doi: 10.3109/15563650.2012.718783

Seifirad, S., and Masoudkabir, F. (2013). Apelin could reduce risk of contrast-induced nephropathy in patients with congestive heart failure. Med. Hypotheses 81, 898-900. doi: 10.1016/j.mehy.2013.08.001

Shahabi, S., Hassan, Z. M., Mahdavi, M., Dezfouli, M., Rahvar, M. T., Naseri, M., et al. (2008). Hot and Cold natures and some parameters of neuroendocrine and immune systems in traditional Iranian medicine: a preliminary study. J. Altern. Complement. Med. 14, 147-156. doi: 10.1089/acm.2007.0693

Shiels, M. S., Albanes, D., Virtamo, J., and Engels, E. A. (2011). Increased risk of lung cancer in 
men with tuberculosis in the alpha-tocopherol, beta-carotene cancer prevention study. Cancer Epidemiol. Biomarkers Prev. 20, 672-678. doi: 10.1158/1055-9965.EPI-10-1166

Strick, R., Strissel, P. L., Borgers, S., Smith, S. L., and Rowley, J. D. (2000). Dietary bioflavonoids induce cleavage in the MLL gene and may contribute to infant leukemia. Proc. Natl. Acad. Sci. U.S.A. 97, 4790-4795. doi: 10.1073/pnas.070061297

Wang, C. R., Nguyen, J., and Lu, Q. B. (2009). Bond breaks of nucleotides by dissociative electron transfer of nonequilibrium prehydrated electrons: a new molecular mechanism for reductive DNA damage. J. Am. Chem. Soc. 131, 11320-11322. doi: $10.1021 /$ ja902675g

Watson, J. (2013). Oxidants, antioxidants and the current incurability of metastatic cancers. Open Biol. 3:120144. doi: 10.1098/rsob.120144

Weel, E. A., Redekop, W. K., and Weening, R. S. (1996). Increased risk of malignancy for patients with chronic granulomatous disease and its possible link to the pathogenesis of cancer. Eur. J. Cancer 32A, 734-735. doi: 10.1016/0959-8049(95)006273

Yang, G. Y., Liao, J., Kim, K., Yurkow, E. J., and Yang, C. S. (1998). Inhibition of growth and induction of apoptosis in human cancer cell lines by tea polyphenols. Carcinogenesis 19, 611-616. doi: 10.1093/carcin/19.4.611

Yang, G. Y., Liao, J., Li, C., Chung, J., Yurkow, E. J., Ho, C. T., et al. (2000). Effect of black and green tea polyphenols on c-jun phosphorylation and $\mathrm{H}(2) \mathrm{O}(2)$ production in transformed and nontransformed human bronchial cell lines: possible mechanisms of cell growth inhibition and apoptosis induction. Carcinogenesis 21, 2035-2039. doi: $10.1093 / \mathrm{carcin} / 21.11 .2035$

Conflict of Interest Statement: The authors declare that the research was conducted in the absence of any commercial or financial relationships that could be construed as a potential conflict of interest.

Received: 15 April 2014; accepted: 13 June 2014; published online: 14 July 2014.

Citation: Seifirad S, Ghaffari $A$ and Amoli MM (2014) The antioxidants dilemma: are they potentially immunosuppressants and carcinogens? Front. Physiol. 5:245. doi: 10.3389/fphys.2014.00245

This article was submitted to Oxidant Physiology, a section of the journal Frontiers in Physiology.

Copyright (c) 2014 Seifirad, Ghaffari and Amoli. This is an open-access article distributed under the terms of the Creative Commons Attribution License (CC BY). The use, distribution or reproduction in other forums is permitted, provided the original author(s) or licensor are credited and that the original publication in this journal is cited, in accordance with accepted academic practice. No use, distribution or reproduction is permitted which does not comply with these terms. 